\title{
Tecnología y Género: \\ La Invisibilidad de lo Femenino en la Construcción de Subjetividades
}

\author{
Technology \& Gender: \\ The Invisibility of the Feminine in the Construction of Subjetivities
}

\author{
Yasna Valenzuela Román \\ Universidad de Talca
}

(Recepción: Marzo 2003 - Aceptación: Julio 2003)

\begin{abstract}
El artículo busca explicitar uno de los trabajos referidos a una línea de investigación más amplia, cual es la relación entre psicología y tecnología. Se busca, desde la mirada de género, mostrar una relación entre tecnología y construcción de subjetividades, no desde el punto de vista del poder sino considerando la ausencia del discurso de lo femenino en la pregunta por el significado, las prácticas, los instrumentos y las lógicas de la tecnología.

Los estudios de género tienden a ver la ausencia de lo femenino en un nivel plasmado de ideologías; las prácticas, en lugar de buscar dicha omisión en su propia constitución conceptual.

Palabras clave: Tecnología, subjetividad, género.
\end{abstract}

\begin{abstract}
The article looks to clarify one of the referred to works in a more expansive field of investigation, which is the relation between Psychology and Technology. It looks, from the point of view of the gender, to show a relation between technology and the construction of subjectivities; not from the aspect of power but in considering the absence of the feminine speech in the question, through the meaning, the use, the instruments, and the logic of technology.

The studies of the gender tend to see the absence of the feminine on a shaped level of ideologies: its use rather than a search for such an omission in its own conceptual condition.

Key words: Technology, subjectivity, gender.
\end{abstract}

\section{Introducción}

Este trabajo se enmarca en una línea de investigación más amplia cual es la relación entre psicología y tecnología. Lo anterior es relevante, ya que no es posible concebir contemporáneamente al sujeto psicológico fuera de un mundo colonizado por las máquinas, representando ellas su condición de posibilidad. De allí que la relación género/tecnología sea fundamental en la construcción de subjetividades. Por tanto, la premisa central busca describir algunas de las consecuencias que el marco actual de las transformaciones tecnológicas tiene con relación al sujeto; ya que el vínculo sujeto/máquina en cuanto

Psicóloga, Licenciada en Psicología, Universidad de Chile. Magister en Psicología, mención Social y de la Personalidad, Universidad Federal de Rio de Janeiro. PhD en Filosofía, mención Epistemología de las Ciencias Sociales, Universidad de Chile. PhD (c) en Psicología, Universidad de Chile.

Correspondencia: Yasna Valenzuela R. Escuela de Psicología, FACE, Universidad de Talca. 2 Norte 685, Talca-Chile. Fono: (56-71) 201 567. E-mail: yvalenzuela@utalca.cl fuente productora de subjetividades constituye un desafío para las ciencias sociales en su conjunto.

El artículo busca explicitar una relación entre tecnología y construcción de subjetividades. Si consideramos que no existe humanidad, civilización sin tecnología y que ésta como fenómeno social hoy sólo tiende a expandirse, se hace imprescindible comprender dicha relación para poder explicar como se conforma el sujeto que está a la base de nuestras disciplinas.

Muchas teorías que intentan explicar la relación género/tecnología lo han hecho desde el punto de vista del poder $^{1}$. Por el contrario en este trabajo, sólo se pretende poner en relieve la cuestión de la importancia de la tecnología en la creación de subjetividades, y por tanto la ausencia de lo femenino en la creación de tecnología, no en términos reproductivos, sino de crea-

1 Por ejemplo: COCKBURN, Cynthia. "TECHNOLOGY, PRODUCTION AND POWER”. En: KIRKUP, Gill y KELLER, Smith (Edit.) "INVENTING WOMEN. SCIENCE, TECHNOLOGY AND GENDER”. Polity Press. Cambridge, 1992. pp. 196-223. 


\section{VALENZUELA}

ción, implica que no participa en la creación de dichas subjetividades, pero a la vez un producto de la misma técnica existente. Así, lo importante de la relación género/tecnología aquí expuesta, no es el número de mujeres en la ciencia y en la técnica (relación ciencia saber técnico ${ }^{2}$ ) como réplica de lo "masculino", sino la pregunta por el significado, las prácticas, los instrumentos y las lógicas de la tecnología, contenga en su discurso la voz del género.

\section{Tecnología como constructora de subjetividades}

La producción de subjetividad se encuentra sobredeterminada multicausalmente. Más aún, podríamos decir que existen diversas dimensiones, diferentes ámbitos donde se recrean los nexos de interdependencia de la subjetividad con el contexto. Sin embargo, esto no quiere decir que cualquier subjetividad emerja con igual fuerza. Existen, por así decirlo, locus privilegiados donde estas subjetividades se moldean.

Uno de ellos, es una cierta revisitación del ideario de oposición hombre/máquina donde este nexo es visto peligrosamente y donde la concepción maquínica queda reducida y opuesta frente a la figura de lo humano. En este sentido, este prisma ayudaría a la continuación de un imaginario dual, algo así como viejas leyendas para un mundo nuevo. Con ello, se producirán dos consecuencias básicas; la reducción de la complejidad social en versiones unívocas y el no-reconocimiento, tanto de la ambigüedad paradjxal de la relación Sujeto/Máquina como su necesaria triangulación entre sujetos en el ámbito de la interactividad.

En las últimas dos décadas, ha tomado consistencia en ciertos medios intelectuales, sobre todo en aquellos conocidos genéricamente bajo la denominación de humanidades, una cierta concepción de la técnica como algo fundamentalmente extraño al hombre. Allí, las máquinas y las maneras de producción que las ponen en movimiento serian, sin lugar a duda, "algo que el propio hombre produce, algo que amplia y extiende sus sentidos y su capacidad de com-

2 HABERMAS, Jürgen. "PROGRESO TÉCNICO Y MUNDO SOCIAL DE LA VIDA". En: "CIENCIA Y TÉCNICA COMO IDEOLOGÍA”. Editorial Rei. México, 1993. pp. 113-129. prensión pero, al mismo tiempo, algo que se le escapa como arena entre los dedos y en lo cual él ya no se puede reconocer"3.

Asimismo, toda una literatura de ficción trae la caricatura de esa idea bastante diseminada según la cual, la proliferación de máquinas cada vez más potentes, versátiles e inteligentes estaría colocando en peligro la propia identidad humana. Como lo sostiene Couchot "ese sentimiento es tanto o más doloroso si concebimos al hombre como un animal desnudo, desprovisto de cualquier otra prótesis instrumental que no sea su lenguaje, el único que fundaría su humani$\mathrm{dad}^{\text {"4. }}$. Ahora bien, fuera del lenguaje -que no deja de ser una tecnología, incluso tal vez la más avanzada de todas las practicadas por el hombre- todas las demás prótesis de acción o percepción humana serian encaradas como artificiales y, por consecuencia, excluidas del universo de la cultura en cuanto territorio de lo humano.

Esta revisitación de posicionamientos clási$\cos$, de antagonismos hombre/máquina, repercute en una serie de ámbitos, ya sea de las relaciones de trabajo bajo el fantasma de la sustitución, en los mecanismos de comunicación y acceso bajo la forma de la exclusión, en el mercado como posesión o expectativa de consumo, en las calles como instrumento de control y también como imaginarios poblados de miedos en expresiones maquínicas propias del año 2000 , donde si bien este horizonte simbólico encuentra sus raíces en un pasado lejano, el contenido de su inseguridad se plasma en mecanismos tecnológicos de última generación. Ejemplos de esto encontramos diseminados en diversos espacios: miedo de que las personas no se relacionen más cara a cara sino solamente a través de Internet, miedo de que los avances sean tan grandes y rápidos que no se pueda acompañarlos, miedo de la excesiva humanización de las máquinas, miedo de que los niños sólo jueguen con máquinas y no desarrollen habilidades sociales, miedo a que el trabajo acabe o quede sólo en las manos de las máquinas.

En todo ello es posible diferenciar los contenidos pero a la vez, identificar una misma lógica binaria y antagónica de argumentación: la oposición entre hombre y máquina. Este tipo de

3 SIMONDON, Gilbert. "DU MODED'EXISTENCEDES OBJETS TECHNIQUÉS". Aubier. París, 1969. p. 61.

4 COUCHOT, Edmond. 'L'ODYSSÉE, MILLEFOISEOU LES MACHINES A LANGAGE”. Traverses. París, 1988. p. 87 . 
pensamiento podría ser calificado de esencialista en dos sentidos: en cuanto se sustenta en una idea de esencia humana predeterminada como opositora de la máquina y también en cuanto constituye parte de un discurso inserto más bien, en un tipo de creencia, de adhesión de fe que en un razonamiento argumental.

De esta forma, dicha oposición se vuelve expresión de un imaginario mítico donde la máquina conspira el tiempo todo contra lo que restaría de esencia en la humanidad. Así, se da lugar a pensar que "en un tiempo y en un lugar en que las máquinas usurpan las actividades creadoras y en las cuales la mediación técnica se vuelve una fuerza generalizante, poco hay para hacer, a no ser filosofar sobre la propia impotencia"s. También es posible concebir a la máquina como locura y engendrar un malestar en términos de la preocupación por la relación del ser humano contemporáneo dudando de la esencia hưmana que saldría incólume de allí. Este malestar con la máquina se sustenta, por otra parte, en la idea de máquinas cada vez mejores con un ser humano de una cualidad cada vez peor, donde las imágenes de esta contradicción están presentes desde el pensamiento teológico al ético-moral.

Lo interesante, en términos de la psicología, es que este imaginario se ha vuelto casi un sentido común extendido y por tanto es posible que algunos individuos se vean y sean vistos como hombres expulsados de un paraíso maquínico, en el sentido de Ciorán: arrojados del tiempo. No sólo es posible pensar lo anterior simbólicamente, la propia dialéctica de la modernización nos proporciona imágenes y datos de los mecanismos maquínico de exclusión.

Este ideario amenazante adquiere ribetes catastróficos en un discurso humanista donde se busca reinstalar una idea de naturaleza en oposición a la idea de lo artificial. El resultado es uniformar la pluralidad, intentar identificar la diversidad de las experiencias, cerrando los ojos para cualquier mecanismo que evidencie la articulación protética de hombres y máquinas o también condenando dichos nexos bajo juicios morales, estéticos o cognitivos.

A su vez, esta relación binaria olvida o reduce, positivizándola, la propia idea de humanis-

s MACHADO, Arlindo. "MÁQUINA E IMAGINÁRIO". Editora da Universidade de São Paulo. São Paulo, 1996. p. 10 . mo. Como sostendrá Savater, le juega una mala pasada a ese humanismo impenitente que configura su propia idea de humanidad en las conexiones indeterminadas con otros ámbitos como lo maquínico ${ }^{6}$. Por tanto, la extrema contradicción es que esa visión unidireccional y esencialista del humanismo, al querer vengarse de la máquina, reduce al propio ser humano que quería enaltecer. De esta forma: "el modo de pensar esencialista, substancialista, es aquél que lleva a tratar las actividades o preferencias propias de ciertos individuos o de ciertos grupos de una cierta sociedad, en un determinado momento, como propiedades sustanciales, inscritas de una vez y para siempre en una especie de 'esencia biológica' o, lo que no es mejor, de una 'esencia cultural', que lleva a los mismos errores en la comparación no con sociedades diferentes pero sí entre períodos sucesivos de uną misma sociedad"?.

De allí que el esencialismo se vuelva ese acuerdo tácito, pre-reflexivo, inmediato, sobre el sentido del mundo que es el fundamento y filtro de las experiencias presentes.

Consecuentemente, al ejercer una mirada positivizada de la relación Sujeto/Máquina, lo anterior también no tiene como hacer emerger los matices, los sesgos, las hibridizaciones, las multicausalidades, las polifonías de dicha relación que precisan ser ecualizadas. En este sentido existe, sin duda, mucho de fetiche en esta actual revisitación de la relación hombre/máquina. A medida que estas últimas se vuelven más atrayentes y amigables, su efecto se tiende a tornar seductor y lisérgico, sobre todo en un público concebido como lo que Bell denomina el hombre sin atributos, para referirse a ese sujeto que en virtud de los procesos tecnológicos y modernizantes ha perdido sus referentes o, como sostendrá Berger, ha quedado transformado en un paria dentro de un mundo sin hogar ${ }^{8}$. Así también las personas se deleitan (y algunas hasta se vician) apretando botones compulsivamente, haciendo zaping o jugando juegos de vi-

6 SAVATER, Fernando. "HUMANISMO IMPENITENTE”. Editorial Anagrama. Barcelona, 1990. p. 15.

7 BOURDIE, Pierre. "RAZÖES PRATICAS: SOBRE A TEORIA DA AÇÃO". Editorial Papirus. São Paulo. 1995. p. 17.

8 Para un análisis mayor al respecto ver: BERGER, Peter y LUCKMANN, Thomas. "MODERNIDAD, PLURALISMO Y CRISIS DE SENTIDO. LA ORIENTACIÓN DEL HOMBRE MODERNO". Editorial Paidós. Barcelona, 1997. 
deo. Los recursos interactivos de que ya disponen gran parte de las actuales máquinas ópticas y acústicas difundidas a nivel de gran público dan un carácter lúdico incluso a la respuesta simple de los movimientos de un operador en la medida que estas aparezcan en una tela bajo la forma de figuras multicoloridas.

Es así como se puede llegar a concebir que "las nanomáquinas además de consagrar una tecnología de los sensores, de los teleespectadores y de los microprocesadores consagraron también un espacio-sin-lugar, un lugar-sin-hogar. Y es, por tales indicadores, que hoy nos encontramos frente a un nuevo dios en que las cualidades de la ubicuidad, de la instantaneidad y de la facultad de ser al mismo tiempo omnipresente y omnividente nos encerró en el mundo de la farsa y donde nosotros mismos, reducidos a ser tan mediáticos como los propios aparatos de mediación, por atrofia, parálisis o incapacidad, no nos interesamos más por proyectar y construir cada momento de la historia"".

Como se ha demostrado, el ideario dual y antagónico hombre y máquina no hace justicia a la descripción de dicha relación compleja y representa una cierta inadecuación de sus categorías conceptuales para intentar seguir el ritmo de alguno de los principales paradoxos que dicha articulación presenta en la sociedad contemporánea. Entre ellos es posible señalar el proceso de agudización de los sentidos, de multisensorialidad logrado por las máquinas en el sujeto a la vez que, justamente en virtud de la naturalización de estos mecanismos, las máquinas se vuelven invisibles haciendo, además, la realidad más inmaterial ${ }^{10}$. Por último, un otro paradoxo posible de explorar se refiere a la doble condición de las máquinas digitales y los aparatos de multimidia en cuanto conjuntamente que despliegan posibilidades de sonido, texto e imagen; unifican la representación sensorial en el código 0 - 1.

9 BRASIL SILVA, Sergio. "REALIDADE DIGITAL: A GLOBALIZAÇÃO EM FANTASIAS". CNPq. Brasilia. 1998. p.5

10 "Como vemos, las cuestiones que se levantan desde los núcleos de especialistas en máquinas ya no son sólo cuestiones de física o de electrónica. Independientemente de su realidad material, las máquinas modernas poseen también una realidad abstracta, de orden lógico, matemático y linguístico, una realidad que puede ser descrita como inmaterial". GANASCIA, Jean-Gabriel. "A INTELIGÊNCIA ARTIFICIAL". Editora Instituto Piaget. Lisboa, 1994. p. 17.
Todo lo dicho puede mostrar, a grandes rasgos, que la matriz de un humanismo radical se cuela, insertándose en visiones de diverso signo político y conceptual, donde se hace preciso una resignificación aguda que de lugar a una crítica efectiva de los mecanismos contemporáneos adoptados por la relación Sujeto/Máquina pero donde no se reduzca lo humano a lo nomaquínico sino que se observe con cuidado las prótesis existentes en las cuáles la máquina hace parte sustantiva de la propia humanidad. De allí que profundizar en la pregunta por la relación genero/tecnología permita resignificar no sólo los espacios de las prácticas sociales, sino reconstruir la propia dimensión de la construcción de la subjetividad por el género.

\section{La Ausencia de lo Femenino en la Tecnología}

Si bien existen muy diversas formas de explicar la ausencia de las mujeres en lo tecnológico, aquí serán reunidas en tres grandes tendencias siguiendo el análisis de López, Santander y González ${ }^{11}$.

a. Feminismo Liberal:

Aquí, se considera neutral a la tecnología y por lo tanto la para que las mujeres puedan estar insertas en ella, sólo es necesario que asuman puestos de poder dentro de esta área. Esto aún no ha ocurrido por dos motivos: no ha transcurrido tiempo suficiente o bien, no existe interés por parte de las mujeres para acceder a ese campo. Es decir, es necesario educar mejor a las niñas en los ámbitos de la ciencia y la tecnología.

\section{b. Ecofeminismo:}

Lo que se plantea es que el modelo cienciatecnología está basado en la dominación y control masculino, en este sentido sería patriarcal. El enfoque plantea que, ya que las mujeres serían más cercanas a la naturaleza, considerar el punto de vista femenino en sistema ciencia-tecnología implicaría un desarrollo más sustentable de éste. Es decir, no se han tenido en cuenta los intereses femeni-

${ }^{11}$ LÓPEZ SEDEÑO, Eulalia, SANTANDER GANA, María Teresa y GONZÁLEZ GARCÍA, Marta. En Mesa Redonda "GÉNERO Y TECNOLOGÍA" que tuvo lugar en la "Primera Conferencia Tecnología para el Desarrollo Humano" 


\section{TECNOLOGÍA Y GÉNERO}

nos en la construcción ni desarrollo de dicho sistema, pero dentro de las mismas lógicas tecnológicas.

\section{c. Perspectiva de Género}

La tecnología es considerada una cultura construida por el patriarcado, lo cual no hace, necesariamente, que sus características sean masculinas por esencia, sino que esta masculinidad depende del periodo histórico en el cual se ha producido. Aquí, se considera la Revolución Industrial como origen ${ }^{12}$ de la separación sexual del trabajo. De allí que a los hombres les corresponda la esfera lo público y a las mujeres la de lo privado.

En las visiones anteriores las problematizaciones son de distinto tipo: en el primer análisis, la respuesta pasa por problematizar el género, en el segundo la tecnología y en el tercero ambas cosas.

Si bien los enfoques expuestos aparecen coherentes, fundamentados y por lo tanto explicativos, es necesario proponer aquí un cuanto punto de vista, que si bien se acerca a la visión de la Perspectiva de Género, pretende problematizar desde la importancia de la tecnología en la creación de subjetividades.

Lo que se quiere agregar es que, partiendo de una idea de tecnología como constituida y constituyente de relaciones sociales, expresa un mundo. Es decir, la tecnología, inscrita como el espacio de posibilidad del dominio de la naturaleza (a nivel de transportes, de elementos de guerra, de avances médicos, de confort humano, de cambio en los modos de saber, de comunicación, del tiempo libre) emerge como un locus sustantivo de lo humano. Pero la liberación de ese dominio involucró un impacto en los principios organizadores de la sociedad y en la conciencia del sujeto, de allí que se corresponda con el nacimiento de las ciencias sociales (entre ellas la psicología) como la posibilidad parcial y múltiple de dar cuenta de esta contradicción entre libertad y dominio, o mejor entre creación de subjetividades y sujeción a ésta ${ }^{13}$. Lo que se

${ }^{12} \mathrm{El}$ origen (Ursprung, en Walter Benjamin), a diferencia de la idea de génesis, permite mirar otra vez, de forma nueva, permite recuperar las prácticas, hacer surgir nuevos enfoques y nuevas prácticas sociales.

13 VALENZUELA ROMÁN, Yasna. "RELACIÓN SUJETO/ TECNOLOGÍA: Algunas Consideraciones Epistemológicas para la Psicología Contemporánea". Tesis para optar al Grado de Doctor en Filosofía, Mención Epistemología de las Ciencias Sociales. Universidad de Chile, Mayo 2001. quiere resaltar es que la tecnología no está referida solamente a lo instrumental sino que es una práctica universal y no neutral, pero sobre todo una expresión del mundo social.

La postura a presentar quiere destacar como los estudios acerca de la relación entre género y tecnología tienden a distinguir desigualdades al nivel de las prácticas y de los usos, pero aún para ellos permanece invisible la idea de la constitución misma de la técnica ${ }^{14}$. Es decir, se tiende a ver la ausencia de lo femenino en un nivel diferente y ya plasmado de ideologías, las prácticas, en lugar de buscar dicha omisión en la propia construcción de subjetividades. Es decir, la subjetividad que la tecnología sin lo femenino ha creado, lleva a asumir que la tecnología depende de sus prácticas y no de su propia constitución. $\mathrm{O}$, si está relacionada con la creación, esta es a nivel reproductivo y no en la implantación de nuevas lógicas, de allí que la tecnología se vuelva "ideología".

Por ejemplo, Abramo ${ }^{15}$ propone que la introducción de tecnología (en sus diferentes etapas de implementación) ayudarían a disminuir la segmentación por género en el mercado laboral. Esto porque al disminuir la utilización de la fuerza física, considerada típicamente masculina, se favorecería el trabajo de las mujeres. Lo anterior ocurre porque entran a participar en las distintas áreas de la producción otro tipo de fortalezas como la flexibilidad técnica, la organización de las nuevas formas de producción, las nuevas concepciones de gestión empresarial, la mayor utilización de tiempo parcial y a domicilio, etc. Sin embargo todo ello "incorpora" a las mujeres a una lógica tecnológica ya existente o en creación de la cual ella no necesariamente participa, con lo cual si bien se gana inserción en esfera pública y tecnológica, la capacidad de enunciación de la tecnología, y por lo tanto el poder de creación de subjetividades continúa en las mismas manos.

\footnotetext{
${ }^{14}$ Para un mayor análisis remitirse a: HABERMAS, Jürgen. “CIENCIA Y TÉCNICA COMO IDEOLOGÍA". Editorial Rei. México, 1993.

15 ABRAMO, Laís. "RECONVERSIÓN PRODUCTIVA, CAMBIO TECNOLÓGICO Y EMPLEO FEMENINO EN AMÉRICA LATINA". En: "REPERCUSIONES DE LA RECONVERSIÓN PRODUCTIVA Y DEL CAMBIO TECNOLÓGICO SOBRE EL EMPLEO Y LAS CONDICIONES DE TRABAJO DE LA MUJER". Oficina Internacional del Trabajo. Documentación base e Informe Final del Seminario Tripartito Latinoamericano. Santiago de Chile. Marzo, 1993. p. 3 y ss.
} 
Lo anterior es relevante ya que si se parte de la base de incorporación a la tecnología como uso, las políticas de no-discriminación, educación e implementación de cambios sociales propuestas irán en ese sentido, con lo cual se continuará repitiendo un modelo tecnológico en el cuál lo femenino es sólo un invitado cuya asistencia no deja marcas.

Por otra parte, el estudio de Rodríguez ${ }^{16}$, reconoce en la Nuevas Tecnologías Informatizadas un cambio en la noción de riesgo en el trabajo, por cuanto se reduce la posibilidad e sufrir efectos no deseados en la ejecución de muchas tareas antes consideradas riesgosas. Durante mucho tiempo salvaguardó la salud femenina excluyéndola como grupo de trabajo en tareas consideradas peligrosas el problema aquí era la discriminación, pero desde el año 1985 se legisla integralmente a favor de la salud en hombres y mujeres. La existencia de una doble jornada laboral para las mujeres, provoca que éstas se vean expuestas a una sumatoria de actividades que provocan nuevos problemas de salud. Así, su no-participación en la creación de lógicas tecnológicas no resuelven los problemas propios de lo femenino en cuanto al trabajo, como por ejemplo, las lesiones por esfuerzos repetitivos, el estrés laboral, así como el síndrome de burn-out propio de actividades como la educación, atención primaria en salud, atención de público, etc. consideradas esencialmente femeninas.

En otro ámbito, Gill y Grint ${ }^{17}$ plantean como una necesidad recuperar a las inventoras y tecnólogas de modo de deshacer la naturalización del vínculo entre masculinidad y tecnología ya que este es un vínculo ideológico y sobre todo exagerado en cuanto remarca y generaliza una supuesta incompetencias técnica de las mujeres. Este argumento ha sido tomado por grupos de feministas para rescatar la contribución

${ }^{16}$ RODRÍGUEZ, Carlos Anibal. "SEGURIDAD Y SALUD DE LA MUJER FRENTE ALA RECONVERSIÓN PRODUCTIVA, CAMBIO TECNOLÓGICO". En: "REPERCUSIONES DE LA RECONVERSIÓN PRODUCTIVA Y DEL CAMBIO TECNOLÓGICO SOBREEL EMPLEO Y LAS CONDICIONES DE TRABAJO DELA MUJER". Oficina Internacional del Trabajo. Documentación base e Informe Final del Seminario Tripartito Latinoamericano. Santiago de Chile. Marzo, 1993. p. 87 y ss.

${ }^{17}$ GILL, Rosalind y GRINT, Keith. "THE GENDERTECHNOLOGY RELATION: CONTEMPORARY THEORY AND RESEARCH". Taylor \& Francis Publishers. Londres 1995. p. 1 y ss. femenina a la innovación tecnológica que ha permanecido oculta en la historia.

Lo que se afirma es que las técnicas creadas $\mathrm{y}$ usadas principalmente por mujeres quedan excluidas del acopio tecnológico. El punto aquí no es hacer el rescate de lo tecnológico desde la mirada oficial, sino el centrar la discusión en el significado de la técnica sobre el cual se sustentará el discurso tecnológico. En palabras de Heidegger ${ }^{18}$ la pregunta por la técnica no es técnica ya que implica un desocultamiento, porque si «el preguntar es la devoción del pensar», se hace necesaria una reflexión no sólo por el significado de la tecnología sino también de sus implicancias ya que la técnica esta unida al conocer en el más amplio sentido. Este planteamiento y no la problematización en cuanto a la contribución de género a la misma tecnología permitirá una mirada femenina visible.

Lo que se propone en este texto por tanto, es que cualquier estudio sobre la relación entre género y tecnología debe tener en cuenta la importancia de la tecnología en el proceso de creación de subjetividades más que en la participación mayor o menor en una tecnología ya creada. Es decir se debe profundizar en el por qué de la dificultad de lo femenino para reconocer sus propias lógicas tecnológicas y por consiguiente que éstas permanezcan en la invisibilidad.

Sólo la pregunta por la existencia de una lógica técnica propiamente femenina no reproductora y sus implicancias en la tecnología como conjunto permitirá que esta vía de construcción de subjetividades sea creada en términos más equitativos entre los géneros. Así, la idea es profundizar en la discusión acerca del tipo de técnica necesaria a nivel socieltal que permita constituir futuras subjetividades sobre las cuales crear y recrear nuevas tecnologías donde sí se encuentren incluidas las subjetividades de lo femenino.

En nuestro presente colonizado casi totalmente por las máquinas y su consiguiente racionalidad instrumental, le corresponde a la tecnología, como discurso de la técnica, no sólo evidenciar y re-crear las relaciones entre los hombres sino también buscar el camino de las igualdades sociales, las cuales no son posibles si la subjetividad de género permanece invisible en un discurso tan importante como es hoy la tecnología.

\footnotetext{
${ }^{18}$ HEIDEGGER, Martin. "CIENCIA Y TÉCNICA". Editorial Universitaria. Santiago, 1993. pp. 47-108.
} 


\section{TECNOLOGÍA Y GÉNERO}

\section{Referencias}

Bourdieu, Pierre. "Razões praticas: sobre a teoria da ação". Editorial Papirus. São Paulo. 1995.

Brasil Silva, Sergio. "Realidade digital: a globalização em fantasias". CNPq. Brasilia. 1998.

Couchot, Edmond. "L'odyssée, mille foise ou les machines a langage". Traverses. París, 1988.

Ganascia, Jean-Gabricl. "A inteligência artificial”. Editora Instituto Piaget. Lisboa, 1994.

Gill, Rosalind y Grint, Keith. "The gender-technology relation: contemporary theory and research". Taylor \& Francis Publishers. Londres 1995.

Habermas, Jürgen. "Ciencia y técnica como ideología". Editorial Rei. México, 1993.

Heidegger, Martin. "Ciencia y técnica". Editorial Universitaria. Santiago, 1993.

Kirkup, Gill y Keller, Smith (Edit.) "Inventing women. science, technology and gender". Polity Press. Cambridge, 1992.

Machado, Arlindo. "Máquina e imaginário". Editora da Universidade de São Paulo. São Paulo, 1996.

Oficina Internacional del Trabajo. "Repercusiones de la reconversión productiva y del cambio tecnológico sobre el empleo y las condiciones de trabajo de la mujer". Documentación base e Informe Final del Seminario Tripartito Latinoamericano. Santiago de Chile. Marzo, 1993.

Savater, Fernando. "Humanismo impenitente". Editorial Anagrama. Barcelona, 1990.

Simondon, Gilbert. "Du mode d'existence des objets techniqués". Aubier. París, 1969.

Valenzuela Román, Yasna. "Relación sujeto/tecnología: Algunas Consideraciones Epistemológicas para la Psicología Contemporánea". Tesis para optar al Grado de Doctor en Filosofía, Mención Epistemología de las Ciencias Sociales. Universidad de Chile, Mayo 2001. 


\title{
La Psicología a Contraluz: Debate Epistemológico y Estatus Disciplinar
}

\section{Psychology in the Light: An Epistemological and Disciplinary Status Debate}

\author{
Mario Villalobos Kirmayr \\ Universidad Santo Tomás de Chile
}

(Recepción: Abril 2003 - Aceptación: Julio 2003)

\begin{abstract}
A través de nociones epistemológicas clásicas como falsación, paradigma y ciencia normal, se examina el estatus científico de la disciplina psicológica tanto en sus niveles de lógica investigativa como de desarrollo institucional. Asimismo, bajo la noción de determinismo estructural maturaniana, se analiza la posibilidad de entender lo mental como objeto de estudio científico.

El examen muestra las dificultades de enmarcar a la psicología dentro del campo de las ciencias, sea por la lógica no falsacionista de su investigación, por el carácter preparadigmático de su institucionalidad, o por la imposibilidad de constituir su objeto de estudio como una unidad con determinación estructural.

Palabras clave: Demarcación científica, paradigma, determinismo estructural.
\end{abstract}

\begin{abstract}
Through classic epistemological notions, such as falsification, paradigm, and traditional science, the scientific status of the psychological discipline is examined as much in its levels of investigative logic as in those of institutional development. At the same time, under Maturana's notion of structural determinism, the possibility of understanding the mental as an object or scientific study is analyzed.

The test shown the difficulties of placing Psychology within a field of sciences; be it for the non-falsifying logic of its investigation, the pre-paradigmatic character of its institutionalism, or for the impossibility of constructing its objective of study as a unit with structural determination.
\end{abstract}

Key words: Scientific demarcation, paradigm, structural determinism.

\section{Introducción}

Las implicancias que el debate epistemológico pasado y actual pueda tener para el presente y futuro de la disciplina psicológica, es una cuestión que pocas veces se oye discutir al interior de la disciplina misma, y que queda más bien como tema de reflexión para filósofos y epistemólogos, salvando las excepciones de figuras como Watzlawick, Von Graserfeld, Piaget, y otros. No obstante dicha despreocupación, y más allá o más acá de cualquier discusión epistemológica, sabemos que tradicionalmente la psicología se ha enmarcado a sí misma dentro de las así llamadas disciplinas científicas; por de pronto, entendiendo por éstas el conjunto de tradiciones de conocimiento que tienen como

Psicólogo, Licenciado en Psicología, Pontificia U. Católica de Chile.

Correspondencia: Mario Villalobos K. Escuela de Psicología, Universidad Santo Tomás de Chile. Avda. Ejército Libertador 146, Santiago-Chile. (56-2) 632 4888,

E-mail: mariovilobos@hotmail.com criterio de demarcación un modo exclusivo y particular de validación, a saber, la validación científica. Si la demarcación provisoria dada para las ciencias incomoda por su apariencia tautológica, consideraré aquello como un buen comienzo para el siguiente debate, pues veremos prontamente que la univocidad para demarcar lo científico, y por extensión lo científicamente psicológico, dista mucho de ser un anhelo siquiera medianamente alcanzable.

Si tomamos a la psicología en conjunto y la sometemos a sólo algunos de los distintos análisis epistemológicos que se han llevado a cabo en esta extensa tradición de debate, podemos obtener como resultado una suerte de informe o perfil disciplinar parcial y tentativo, que pese a su carácter preliminar, nos puede aportar una visión diferente y complementaria a -aunque no necesariamente armónica o congruente con- la que nosotros hacedores de la psicología solemos tener.

Para ello me centraré en tres aproximaciones epistemológicas que tienen orígenes, discur- 